\title{
Switching to Aripiprazole as a Strategy for Weight Reduction: A Meta-Analysis in Patients Suffering from Schizophrenia
}

\author{
Yoram Barak and Dov Aizenberg \\ Abarbanel Mental Health Center and Geha Mental Health Center Affiliated with the Sackler Faculty of Medicine, Tel-Aviv University, \\ Bat-Yam 59100, Israel \\ Correspondence should be addressed to Yoram Barak, mdybarak@gmail.com
}

Received 17 April 2010; Revised 15 July 2010; Accepted 28 July 2010

Academic Editor: S. B. Heymsfield

Copyright ( $\odot 2011$ Y. Barak and D. Aizenberg. This is an open access article distributed under the Creative Commons Attribution License, which permits unrestricted use, distribution, and reproduction in any medium, provided the original work is properly cited.

\begin{abstract}
Weight gain is one of the major drawbacks associated with the pharmacological treatment of schizophrenia. Existing strategies for the prevention and treatment of obesity amongst these patients are disappointing. Switching the current antipsychotic to another that may favorably affect weight is not yet fully established in the psychiatric literature. This meta-analysis focused on switching to aripiprazole as it has a pharmacological and clinical profile that may result in an improved weight control. Nine publications from seven countries worldwide were analyzed. These encompassed 784 schizophrenia and schizoaffective patients, $473(60 \%)$ men and $311(40 \%)$ women, mean age $39.4 \pm 7.0$ years. The major significant finding was a mean weight reduction by $-2.55 \pm 1.5 \mathrm{kgs}$ following the switch to aripiprazole $(P<.001)$. Switching to an antipsychotic with a lower propensity to induce weight gain needs be explored as a strategy. Our analysis suggests aripiprazole as a candidate for such a treatment strategy.
\end{abstract}

\section{Introduction}

Excessive bodyweight gain is not a new clinical observation amongst patients treated with antipsychotics and was already reported during the 1950's as an adverse effect of typical antipsychotic drug treatment. Hence, one of the major difficulties facing patients suffering from schizophrenia and health care professionals is obesity. In comparison to the general population, these patients have higher rates of morbidity and mortality, which are thought to be due, in part, to increased rates of obesity. This problem has been exacerbated more recently with the introduction and increased use of second generation antipsychotics (SGA), several of which are associated with the risk of weight gain and metabolic disturbance [1]. The magnitude of bodyweight gain reported in the largest naturalistic treatment studies (the CATIE study) of patients treated by SGAs with bodyweight gain of $>7 \%$ compared with baseline was in the range of $12 \%$ to $30 \%$ $[2,3]$.

In many obese patients suffering from schizophrenia, treatment based on only lifestyle interventions is limited by the core symptomatology of the disease, medication side effects, and patient's nonadherence and thus may not result in desirable weight loss. Nevertheless, antipsychoticinduced weight gain is an important modifiable parameter in the high rates of obesity in this population. The need for an effective complementary drug treatment would be advantageous specifically for this large group of vulnerable patients.

The Schizophrenia Patient Outcomes Research Team (PORT) psychopharmacological treatment recommendations provide a wide-ranging summary of current evidencebased pharmacological treatment practices. The latest report of the PORT group was recently published, and the pharmacological prevention and treatment of antipsychoticassociated weight gain in schizophrenia were specifically addressed [4]. The PORT approach for obesity associated with antipsychotic medications is important in treatment and prevention. Three pharmacological strategies have been evaluated by this group: (1) switching current antipsychotic to an antipsychotic with a lower propensity for weight gain, (2) employing a medication to prevent weight gain, and (3) 
addition of a medication to patients who have gained weight during therapy in order to promote weight loss. Regrettably, there is currently insufficient evidence to recommend any of these strategies.

The equivocal recommendations of the PORT group have been challenged by researchers and clinicians who have focused on "switching" antipsychotic medications as a viable strategy to induce weight loss. Development of obesity in patients suffering from schizophrenia who were stabilized on SGA has been the framework by these researchers. Risks and benefits of staying on current treatment versus switching to another agent and switching strategies were evaluated, concluding that switching to another antipsychotic with more favorable side effects is called for if other weight-loss strategies fail [3]. However, there is little evidence to support which is the best switching strategy [5]. There are several SGAs that may be feasible candidates for switching strategy. These may include ziprasidone, amisulpride, paliperidone, and aripiprazole [6]. Although the debate about the potential relationship between aripiprazole therapy and the risk of weight gain is not yet resolved, it has been also stated that this specific aspect of the safety profile of aripiprazole assumed on the basis of its unusual pharmacological characteristics may result in a decreased adverse impact on weight that often complicates management for a large number of patients suffering from schizophrenia treated chronically with antipsychotics [7, 8]. Clinical characteristics of aripiprazole in comparison to other SGAs have been recently evaluated by the Cochrane Collaboration. The authors observed that aripiprazole was associated with fewer side effects such as weight gain, cholesterol increase, sedation, and prolactinassociated side effects, concluding that aripiprazole may be more tolerable in terms of metabolic effects and sedation [9].

The primary objective of this meta-analysis was to examine the efficacy of switching to aripiprazole as a strategy for achieving weight loss in schizophrenia patients currently using other antipsychotics.

\section{Materials and Methods}

2.1. Study Selection and Data Collection. At the beginning of this project, we drafted a study protocol. For our analysis, we included all trials in which patients suffering from schizophrenia or schizoaffective disorder (DSM-IV criteria) treated with any antipsychotic were switched to aripiprazole monotherapy in acute-phase or maintenance treatment. We excluded placebo groups where present.

The authors independently reviewed references and abstracts retrieved by the search, assessed the completeness of data abstraction, and confirmed quality rating. We used a structured data abstraction form to ensure consistency of appraisal for each study. Investigators were contacted and asked to provide data to supplement the incomplete reporting of the original articles [10].

2.2. Outcome Measures. We defined mean change from baseline weight (and standard deviation) as the primary efficacy outcome measure in the present analysis.
Studies were included only if mean change in weight was reported as well as the following variables: (a) design: industry support, sample size, previous antipsychotic treatment, aripiprazole dose, and treatment duration. (b) demographic: age, gender, and diagnosis.

2.3. PubMed. A PubMed literature search (last search April 2010) was performed to identify all original research articles using aripiprazole to determine weight change in adult patients.

The search terms were ("aripiprazole" or "abilify"), ("schizophrenia" or "schizoaffective disorder") and ("switch", "weight", "metabolic", "BMI", or "obesity") in the abstract, title, or index terms.

2.4. Additional Electronic Searches. Studies were searched for using the following search strategy: Diagnosis = Schizophrenia* or Schizoaffective* ${ }^{*}$ and Intervention $=$ aripiprazole. References were searched using the same search strategy with the addition of Free-Text = weight. The researchers conducted searches on the MEDLINE and PsychInfo and checked various meta-analyses and review articles on the 8th of April 2010.

Searching other resources includes the following.

(1) Hand searches. We also searched the ongoing trial register http://clinicaltrials.gov/ in the USA.

(2) Personal communication. Experts in this field were asked if they knew of any study that met the inclusion criteria of this review.

(3) Reference checking. Reference lists of the included studies, previous systematic reviews, and major textbooks of schizophrenia written in English were checked for published reports and citations of unpublished research

2.5. Data Extraction and Management. Both authors extracted data from the included studies. Again, any disagreement was discussed, and decisions were documented. Retrieved data was rated dichotomously by each author as either "sufficient" or "inadequate". Only studies for which both authors' ratings were "sufficient" were included in the present analysis.

If necessary, we contacted the authors of the studies for clarification. We extracted the following data:

(i) participant characteristics,

(ii) intervention details,

(iii) outcome measures of interest from the included studies.

2.6. Data Synthesis and Statistical Analysis. Our primary method for the assessment of weight reduction was through pooled estimates using fixed-effects meta-analyses methodology. This allowed the integration of information to provide an estimate with a $95 \%$ confidence interval (CI). The results also allow us to make probabilistic statements about the 
effect size; that is, we are able to answer the following question: "Given the observed data, what is the probability that switching treatment to aripiprazole will induce weight loss?"

Analyses were performed on those antipsychotic medications with published studies that were completed in the period from 2003 to 2010. Also, pooled results were estimated by subgroups of diagnosis, sample size, and gender.

Data analysis was carried out with the use of the Statistical Analysis System software, SAS Institute, version 9.1.3 from 2007.

\section{Results and Discussion}

3.1. Results. The literature search yielded 47 articles, of which 11 manuscripts fulfilled the inclusion criteria for the present meta-analysis. These studies spanned the period from 2003 to 2010. Taken altogether, the cumulative sample size included 786 patients [11-21]. There were also 2 case reports $[13,14]$ which were excluded from the final analysis. Characteristics of the final 784 analyzed participants are presented in Table 1.

The sample analyzed was composed mainly of adult males suffering from schizophrenia. Mean age for the sample was $39.4 \pm 7.0$ years (range: 30 to 54 ); there were 473 $(60 \%)$ men and $311(40 \%)$ women, of whom $644(82 \%)$ were diagnosed as suffering from schizophrenia, and 140 $(18 \%)$ were suffering from schizoaffective disorder. Half of the studies were conducted in the USA (5/9), two in Korea, one each in the Netherlands, Japan, and Eastern Europe. It should be noted that one of the studies was an international collaboration between USA and East European researchers. Thus, 422 (54\%) of patients were of Caucasian ethnicity, and 148 (19\%) were Korean.

Antipsychotic medications resulting in weight gain for which the later switch to aripiprazole was undertaken were distributed as follows: olanzapine $352(46 \%)$, risperidone $226(30 \%)$, typical-first generation 168 (22\%), sulpiride $17(3 \%)$, clozapine $11(1 \%)$, and quetiapine $10(1 \%)$. Mean weight for the sample prior to switching to aripirazole was $83.8 \pm 15.4 \mathrm{kgs}$ (range: 63 to 104 ); mean aripirazole dose following the switch was $20.2 \pm 5.0 \mathrm{mgs} /$ daily (range: 15 to 30 ), and mean duration of aripirazole treatment was $26.2 \pm$ 16.9 weeks (range: 8 to 56 ). In all studies herein analyzed, a minimal reduction in weight of $1.2 \mathrm{kgs}$ was reported. In the four largest studies included $[11,12,18,19]$, the reduction in weight was statistically significant, $P<.001$. It is of interest to note that the weight reduction observed in the two case reports ( 10.5 and $16.8 \mathrm{kgs}$ ) was extremely higher than that reported in the large-scale studies $[13,14]$.

Body mass index (BMI) was reported only in 2 studies, and thus we were unable to analyse the significance in it's change. In one study [11], reduction in BMI following aripiprazole treatment was -1.3 , and in the second study [16] it was -0.5 .

Following the switch to aripirazole, a reduction in weight of $7 \%$ or more was observed in 52/484 patients (10.7\%) while

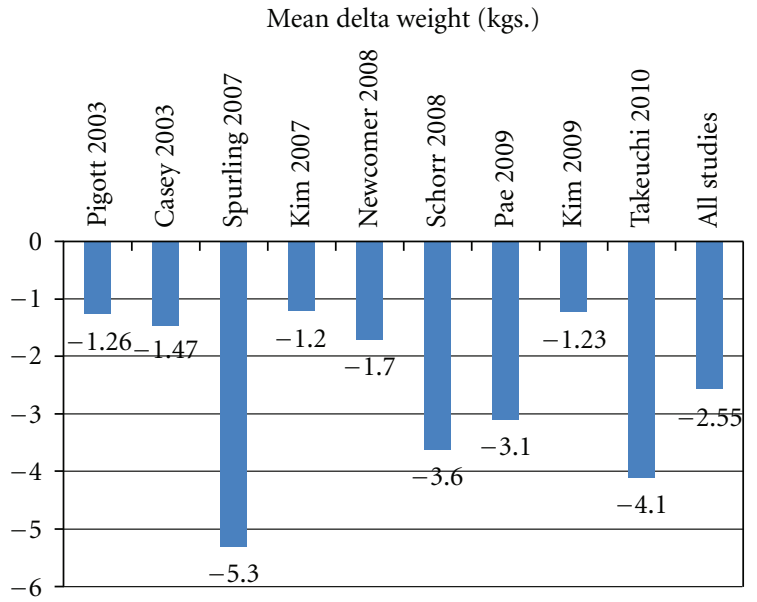

FIGURE 1: Mean reduction in weight (kgs) across analyzed studies.

an increase of $7 \%$ or more in weight was observed in 22/484 (4.5\%); this was highly statistically significant, $P<.0004$.

Correlations computed for weight reduction and gender, age, ethnicity, or aripiprazole dose did not reach statistical significance. Duration of aripiprazole treatment was negatively correlated with the weight change (longer time $=$ greater weight loss) not reaching statistical significance $\left(R^{2}\right.$ $=-0.48, P=.085$ ).

The major significant findings of the present analysis were the following:

(i) mean weight reduction by $-2.55 \pm 1.5 \mathrm{kgs} 95 \% \mathrm{CI}$ : -3.7 to -1.4 (range: -1.2 to -5.3 ) following the switch to aripiprazole was statistically significant, $P<$ .001. See Figure 1.

(ii) mean weight reduction was statistically greater for patients diagnosed as suffering from schizophrenia $(-2.67 \mathrm{kgs})$ when compared to mean reduction in patients suffering from schizoaffective disorder $(-2.18 \mathrm{kgs}), P<.022$.

(iii) the most significant mean weight reduction $(-2.74 \mathrm{kgs})$ was noted in patients who were exposed to olanzapine prior to switching treatment to aripiprazole, $P<.001$.

3.2. Discussion. The introduction of the SGAs has brought to the forefront the awareness that antipsychotic treatment is associated with an increase in weight, adversely affecting cardiac and metabolic risks. However, not all antipsychotics are thought to contribute to the same degree, and the possibility of switching antipsychotic medications to reduce the induced weight gain has been intriguing [22].

Several reports on studies in which already overweight, antipsychotic-treated patients were allocated to switch to aripiprazole have been published. Aripiprazole treatment was then associated with significant improvements in weight. However, to the best of our knowledge, no meta-analysis encompassing all switching studies has been carried out to date. The strength of weight reduction effect was deemed 


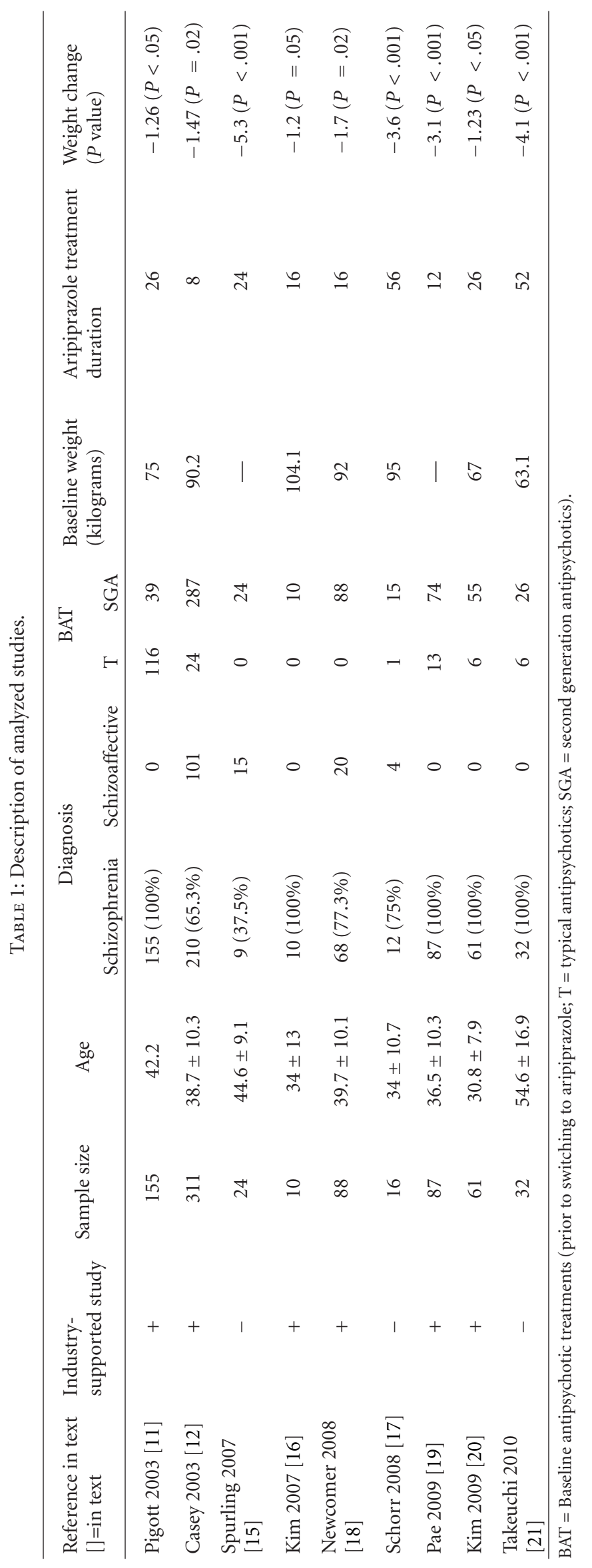


clinically meaningful in the present analysis and is consistent with previous research in weight-unselected patients [23]. Our results support the suggestion that switching might be considered for any antipsychotic-treated patients who had gained weight. More so, for those possessing pre-existing cardiac and metabolic risk factors, an early switch to a more metabolically neutral antipsychotic such as aripiprazole might serve best.

In general, switching studies are carried out to evaluate whether switching results in further symptom improvement [24]. The BETA and EU-BETA studies [25], as well as other switching studies $[12,16,24]$, have focused mainly on the degree of clinical improvement, tolerability, and safety profiles. However, data from these studies was herein analyzed to determine whether weight reduction may be achieved, as clinicians who switch patients from one antipsychotic agent to another are often concerned about the potential for reducing this serious side effect. Nevertheless, some patients gain weight when treatment with an antipsychotic is switched to aripiprazole. A clinically meaningful change in weight is usually defined as a change greater than $7 \%$ of body weight before an intervention [26]. In the Kim et al. naturalistic switch study [27], the authors reported that the rate of patients switched to aripiprazole who had gained weight was lower than that of patients continuing standard-of-care antipsychotics. In the present analysis, the rate of patients who had lost more than $7 \%$ of their weight following the switch to aripiprazole was more than twice that of patients who had gained more than $7 \%$ of their weight prior to the switch.

3.3. Limitations. Some of the analyzed studies lacked data as to $\mathrm{BMI}$ and to the rates of the $7 \%$ change in weight. There were two single cases that were statistically "outliers" in the reported weight reduction and were thus excluded from the final analysis. Age ranges limit generalization of our findings to adolescents or the elderly. Finally, all of the studies with the largest sample size were industrially supported.

\section{Conclusions}

Patients taking antipsychotic medications for psychiatric disorders have also many risk factors for medical comorbidities. There is a pressing need for effective interventions to address problems related to the additional iatrogenic burden from weight gain caused by antipsychotic medications. For patients suffering from schizophrenia, discontinuation of antipsychotic medication is not advisable, and thus switching to an antipsychotic with a lower propensity to induce weight gain needs be explored as a strategy. Our analysis suggests, in accord with previous findings [28], that aripiprazole could be useful for patients treated with other antipsychotics who show significant weight gain, a well-established side effect of many antipsychotics.

\section{Disclosure}

Authors (Y. Barak and D. Aizenberg) have received an unrestricted educational Grant by Biotis Ltd (Israel).

\section{References}

[1] G. Faulkner, T. Cohn, and G. Remington, "Interventions to reduce weight gain in schizophrenia," Cochrane Database of Systematic Reviews, no. 1, Article ID CD005148, 2007.

[2] H. A. Nasrallah, "Metabolic findings from the CATIE trial and their relation to tolerability," CNS Spectrums, vol. 11, no. 7, supplement 7, pp. 32-39, 2006.

[3] T. Baptista, Y. ElFakih, E. Uzcátegui et al., "Pharmacological management of atypical antipsychotic-induced weight gain," CNS Drugs, vol. 22, no. 6, pp. 477-495, 2008.

[4] R. W. Buchanan, J. Kreyenbuhl, D. L. Kelly et al., "The 2009 schizophrenia PORT psychopharmacological treatment recommendations and summary statements," Schizophrenia Bulletin, vol. 36, no. 1, pp. 71-93, 2010.

[5] M. Weber, A. M. Gutierrez, and M. Mohammadi, "The risks and benefits of switching antipsychotics: a case study approach," Perspectives in Psychiatric Care, vol. 45, no. 1, pp. 54-61, 2009.

[6] D. I. Brixner, Q. Said, P. K. Corey-Lisle et al., "Naturalistic impact of second-generation antipsychotics on weight gain," Annals of Pharmacotherapy, vol. 40, no. 4, pp. 626-632, 2006.

[7] S. Gentile, "A systematic review of quality of life and weight gain-related issues in patients treated for severe and persistent mental disorders: focus on aripiprazole," Neuropsychiatric Disease and Treatment, vol. 5, no. 1, pp. 117-125, 2009.

[8] C.-U. Pae, "A review of the safety and tolerability of aripiprazole," Expert Opinion on Drug Safety, vol. 8, no. 3, pp. 373-386, 2009.

[9] K. Komossa, C. Rummel-Kluge, F. Schmid et al., "Aripiprazole versus other atypical antipsychotics for schizophrenia," Cochrane Database of Systematic Reviews, no. 4, Article ID CD006569, 2009.

[10] S. H. Downs and N. Black, "The feasibility of creating a checklist for the assessment of the methodological quality both of randomised and non-randomised studies of health care interventions," Journal of Epidemiology and Community Health, vol. 52, no. 6, pp. 377-384, 1998.

[11] T. A. Pigott, W. H. Carson, A. R. Saha, A. F. Torbeyns, E. G. Stock, and G. G. Ingenito, "Aripiprazole for the prevention of relapse in stabilized patients with chronic schizophrenia: a placebo-controlled 26-week study," Journal of Clinical Psychiatry, vol. 64, no. 9, pp. 1048-1056, 2003.

[12] D. E. Casey, W. H. Carson, A. R. Saha et al., "Switching patients to aripiprazole from other antipsychotic agents: a multicenter randomized study," Psychopharmacology, vol. 166, no. 4, pp. 391-399, 2003.

[13] B. Chavez and R. A. Poveda, "Efficacy with high-dose aripiprazole after olanzapine-related metabolic disturbances," Annals of Pharmacotherapy, vol. 40, no. 12, pp. 2265-2268, 2006.

[14] S.-K. Lin and C.-K. Chen, "Reversal of antipsychotic-induced hyperprolactinemia, weight gain, and dyslipidemia by aripiprazole: a case report," Journal of Clinical Psychiatry, vol. 67, no. 8, p. 1307, 2006.

[15] R. D. Spurling, J. S. Lamberti, D. Olsen, X. Tu, and W. Tang, "Changes in metabolic parameters with switching to aripiprazole from another second-generation antipsychotic: a retrospective chart review," Journal of Clinical Psychiatry, vol. 68, no. 3, pp. 406-409, 2007.

[16] S. H. Kim, O. Ivanova, F. A. Abbasi, C. A. Lamendola, G. M. Reaven, and I. D. Glick, "Metabolic impact of switching antipsychotic therapy to aripiprazole after weight gain: a pilot study," Journal of Clinical Psychopharmacology, vol. 27, no. 4, pp. 365-368, 2007. 
[17] S. G. Schorr, C. J. Slooff, R. Postema et al., "A 12-month follow-up study of treating overweight schizophrenic patients with aripiprazole," Acta Psychiatrica Scandinavica, vol. 118, no. 3, pp. 246-250, 2008.

[18] J. W. Newcomer, J. A. Campos, R. N. Marcus et al., "A multicenter, randomized, double-blind study of the effects of aripiprazole in overweight subjects with schizophrenia or schizoaffective disorder switched from olanzapine," Journal of Clinical Psychiatry, vol. 69, no. 7, pp. 1046-1056, 2008.

[19] C.-U. Pae, A. Serretti, A. Chiesa et al., "Immediate versus gradual suspension of previous treatments during switch to aripiprazole: results of a randomized, open label study," European Neuropsychopharmacology, vol. 19, no. 8, pp. 562570, 2009.

[20] S.-W. Kim, I.-S. Shin, J.-M. Kim et al., "Effectiveness of switching to aripiprazole from atypical antipsychotics in patients with schizophrenia," Clinical Neuropharmacology, vol. 32, no. 5, pp. 243-249, 2009.

[21] H. Takeuchi, H. Uchida, T. Suzuki, K. Watanabe, and H. Kashima, "Changes in metabolic parameters following a switch to aripiprazole in Japanese patients with schizophrenia: one-year follow-up study," Psychiatry and Clinical Neurosciences, vol. 64, no. 1, pp. 104-106, 2010.

[22] T. J. R. Lambert, "Switching to aripiprazole from olanzapine leads to weight loss in overweight people with schizophrenia or schizoaffective disorder," Evidence-Based Mental Health, vol. 12, no. 2, p. 50, 2009.

[23] J. M. Kane, O. Osuntokun, L. A. Kryzhanovskaya et al., "A 28week, randomized, double-blind study of olanzapine versus aripiprazole in the treatment of schizophrenia," Journal of Clinical Psychiatry, vol. 70, no. 4, pp. 572-581, 2009.

[24] R. Tandon, R. N. Marcus, E. G. Stock et al., "A prospective, multicenter, randomized, parallel-group, open-label study of aripiprazole in the management of patients with schizophrenia or schizoaffective disorder in general psychiatric practice: Broad Effectiveness Trial With Aripiprazole (BETA)," Schizophrenia Research, vol. 84, no. 1, pp. 77-89, 2006.

[25] J. Wolf, F. Janssen, H. Lublin et al., "A prospective, multicentre, open-label study of aripiprazole in the management of patients with schizophrenia in psychiatric practice in Europe: Broad Effectiveness Trial with Aripiprazole in Europe (EUBETA)," Current Medical Research and Opinion, vol. 23, no. 10, pp. 2313-2323, 2007.

[26] W. Deberdt, A. Winokur, P. A. Cavazzoni et al., "Amantadine for weight gain associated with olanzapine treatment," European Neuropsychopharmacology, vol. 15, no. 1, pp. 13-21, 2005.

[27] C. Y. Kim, S. Chung, J.-N. Lee et al., "A 12-week, naturalistic switch study of the efficacy and tolerability of aripiprazole in stable outpatients with schizophrenia or schizoaffective disorder," International Clinical Psychopharmacology, vol. 24, no. 4, pp. 181-188, 2009.

[28] P. J. Weiden, "Switching antipsychotics as a treatment strategy for antipsychotic-induced weight gain and dyslipidemia," Journal of Clinical Psychiatry, vol. 68, supplement 4, pp. 3439, 2007. 


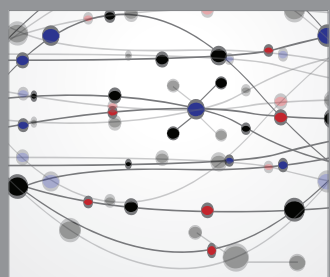

The Scientific World Journal
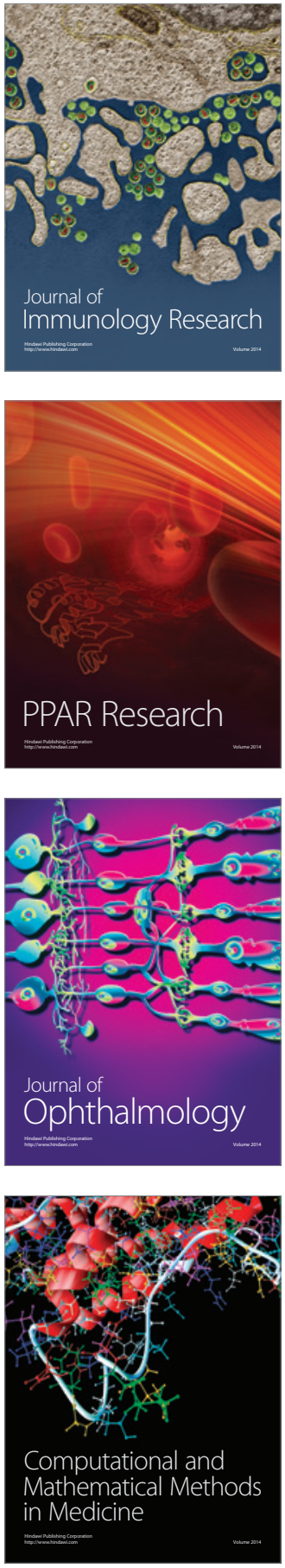

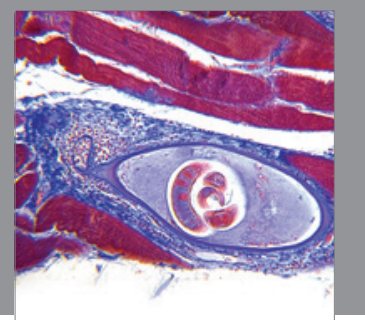

Gastroenterology

Research and Practice
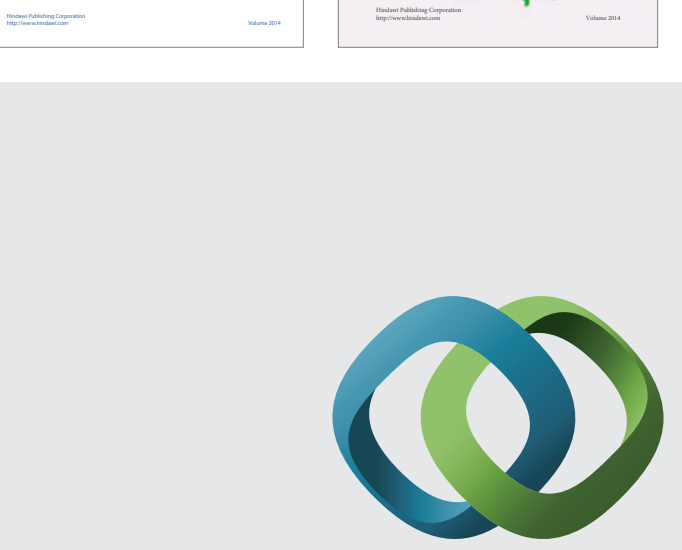

\section{Hindawi}

Submit your manuscripts at

http://www.hindawi.com
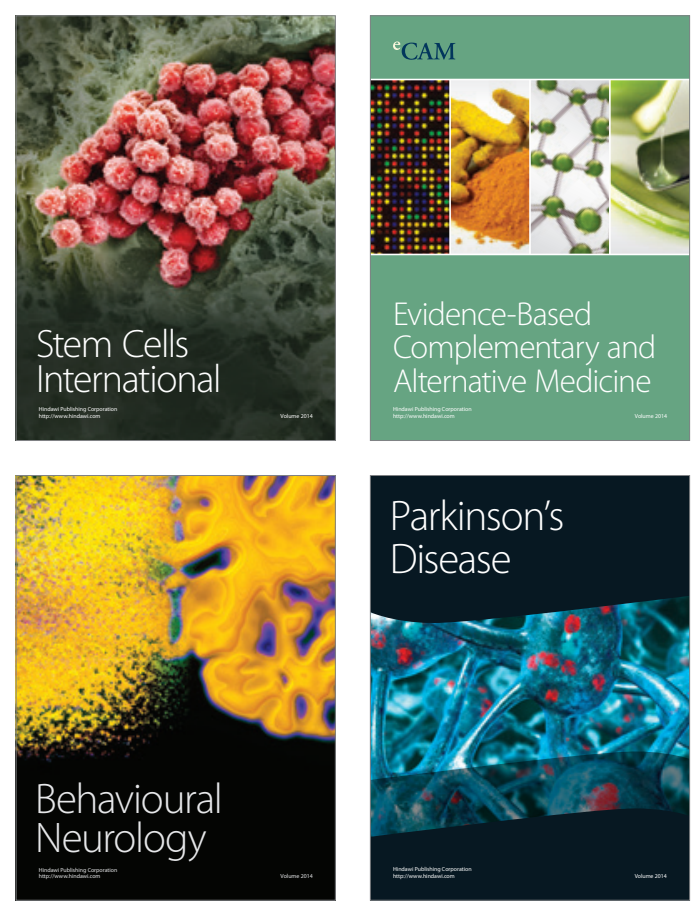

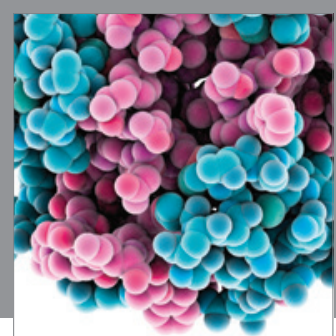

Journal of
Diabetes Research

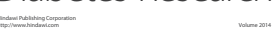

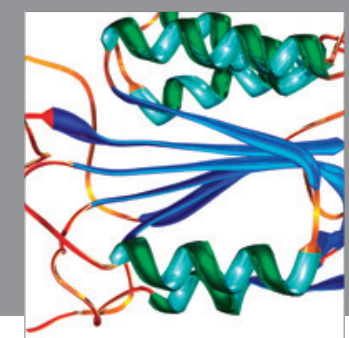

Disease Markers
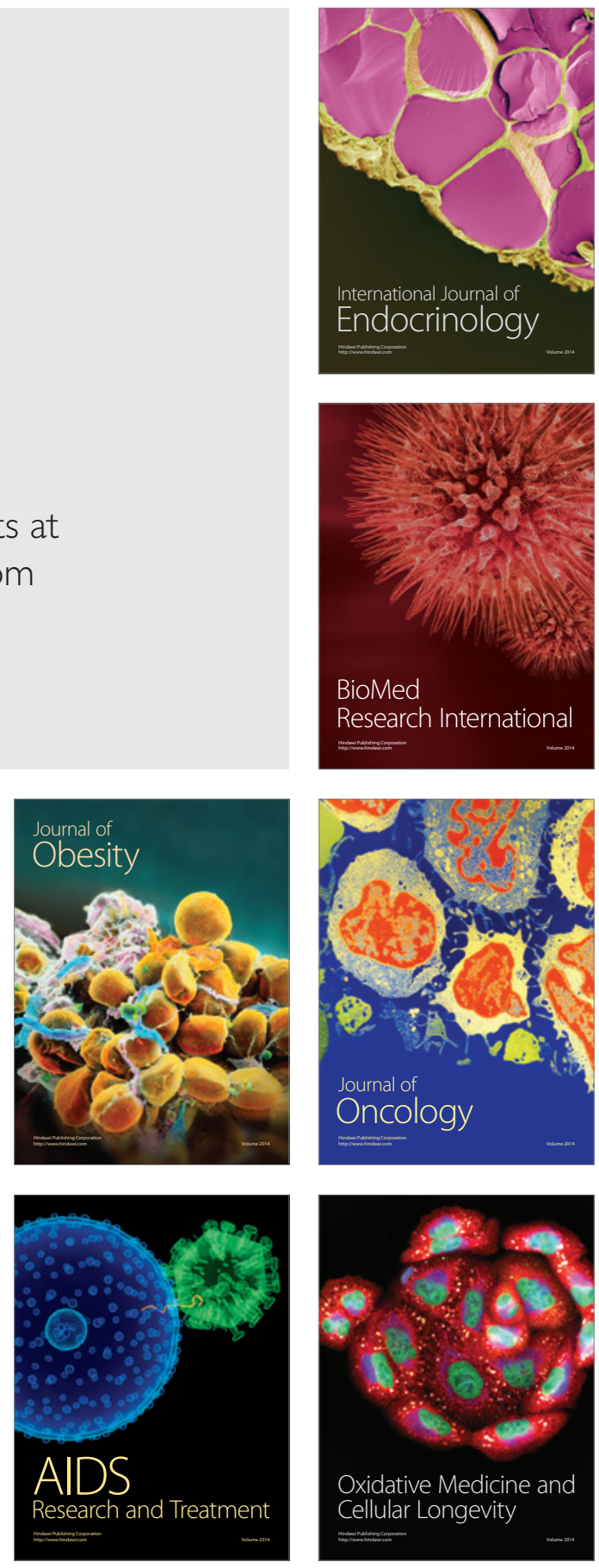